\title{
Counting Periodic Points in Parallel Graph Dynamical Systems
}

\author{
Juan A. Aledo $\mathbb{D},{ }^{1}$ Ali Barzanouni $\mathbb{D},{ }^{2}$ Ghazaleh Malekbala $\mathbb{D}^{2},{ }^{2}$ Leila Sharifan $\left(\mathbb{D},{ }^{2}\right.$ \\ and Jose C. Valverde $\left.{ }^{1}\right)^{1}$ \\ ${ }^{1}$ Department of Mathematics, University of Castilla-La Mancha, Albacete, Spain \\ ${ }^{2}$ Department of Mathematics and Computer Sciences, Hakim Sabzevari University, Sabzevar, Iran
}

Correspondence should be addressed to Jose C. Valverde; jose.valverde@uclm.es

Received 27 March 2020; Accepted 22 May 2020; Published 14 September 2020

Academic Editor: Lucia Valentina Gambuzza

Copyright $\odot 2020$ Juan A. Aledo et al. This is an open access article distributed under the Creative Commons Attribution License, which permits unrestricted use, distribution, and reproduction in any medium, provided the original work is properly cited.

Let $F:\{0,1\}^{n} \longrightarrow\{0,1\}^{n}$ be a parallel dynamical system over an undirected graph with a Boolean maxterm or minterm function as a global evolution operator. It is well known that every periodic point has at most two periods. Actually, periodic points of different periods cannot coexist, and a fixed point theorem is also known. In addition, an upper bound for the number of periodic points of $F$ has been given. In this paper, we complete the study, solving the minimum number of periodic points' problem for this kind of dynamical systems which has been usually considered from the point of view of complexity. In order to do this, we use methods based on the notions of minimal dominating sets and maximal independent sets in graphs, respectively. More specifically, we find a lower bound for the number of fixed points and a lower bound for the number of 2-periodic points of $F$. In addition, we provide a formula that allows us to calculate the exact number of fixed points. Furthermore, we provide some conditions under which these lower bounds are attained, thus generalizing the fixed-point theorem and the 2-period theorem for these systems.

\section{Introduction}

Dynamical systems have a long and brilliant history of applications in biological networks [1], epidemic networks [2], social networks [3], and engineering control systems [4]. In this paper, we deal with a particular class of finite dynamical systems, named deterministic Boolean networks $[5,6]$. A deterministic Boolean network is a time-discrete dynamical system $F:\{0,1\}^{n} \longrightarrow\{0,1\}^{n}$ whose evolution operator is also a Boolean function. These networks were introduced by Kauffman [7] and also studied in works like [8-11] as models of genes activity and interactions.

Besides the applications of Boolean networks in biological and social systems [12], these networks have been used in other branches of science for modeling problems in different fields such as computer science $[13,14]$, chemistry $[15,16]$, mathematics $[17,18]$, and physics $[19,20]$. In fact, due to their versatility, Boolean networks have become a profusely studied topic $[5,6,21-26]$ in the last two decades.

Boolean networks can be represented by means of graphs. Specifically, associated with a (simple undirected) graph $G=(V, E)$ with vertex set $V=\{1, \ldots, n\}$ and a Boolean function $f:\{0,1\}^{n} \longrightarrow\{0,1\}$, we can define a homogeneous dynamical system, denoted by $F$ :

$$
\begin{gathered}
F:\{0,1\}^{n} \longrightarrow\{0,1\}^{n}, \\
F\left(x_{1}, \ldots, x_{n}\right)=\left(y_{1}, \ldots, y_{n}\right),
\end{gathered}
$$

such that each $y_{i}$ is given by the function $f_{i}$ which is the restriction of the global function $f$ to the state of the entity $i$ and its neighbors. The graph $G$ is called the dependency graph of $F$. When all the entities are updated in a synchronous manner, the system is called a parallel dynamical system (PDS) [27-34], while if all the entities are updated in an asynchronous way, the system is called a sequential dynamical system $[5,6,24,35,36]$. When the function $f$ is a maxterm (resp., a minterm), the system is called MAX - PDS (resp., MIN - PDS), in the parallel case, while in the sequential case, it is named MAX-SDS (resp., MIN-SDS). Here, by maxterm (resp., minterm), we mean $f\left(x_{1}, \ldots, x_{n}\right)=z_{1} \vee \cdots \vee z_{n}$ (resp., $f\left(x_{1}, \ldots, x_{n}\right)=z_{1} \wedge \ldots$ $\left.\wedge z_{n}\right)$, where $z_{i}=x_{i}$ or $z_{i}=x_{i}^{\prime}$. 
Following the notations of [33], we shall denote by $W \subseteq V$ (resp., $W^{\prime} \subseteq V$ ) the set of vertices in $V$ such that the corresponding variables in $f$ appear in a direct (resp., complemented) form. Moreover, for each $i \in V$ and $Q \subseteq V$, we define

$$
\begin{aligned}
A_{G}(i) & =\{j:\{i, j\} \in E\}, \\
\overline{A_{G}(i)} & =A_{G}(i) \cup\{i\}, \\
A_{G}(Q) & =\bigcup_{i \in Q} A_{G}(i), \\
\overline{A_{G}(Q)} & =A_{G}(Q) \cup Q .
\end{aligned}
$$

Previous studies in deterministic Boolean (finite) networks (also known as Boolean finite dynamical systems) show that the relationship between the structure of the network and its dynamics is a key issue. In this sense, some authors have set out the dynamical problem of finding fixed points and periodic points (see, for instance, [26, 36-45]). From the point of view of complexity, the problem of finding fixed points (or, more generally, periodic points) of these systems is NP-hard [46, 47]. Related to the problem of counting periodic points, other problems such as the maximum number problem and the minimum number problem are usually considered. In the literature, one can find some works on the study of the maximum number of fixed points of particular classes of Boolean finite dynamical systems (see [37-39]). In particular, in [38, 39], the authors established a relationship between the maximum number of fixed points in a special class of PDS and the maximum number of maximal independent sets. In this paper, we use such maximal independent sets to establish the minimum number of 2-periodic points in homogeneous PDS induced by a maxterm or minterm. Regarding the minimum number of fixed points' problem, in the recent work [48], the authors studied its complexity and established that finding a Boolean network having at least $k$ fixed points is in $P$ or complete for $\mathrm{NP}, \mathrm{NP}^{\#} \mathrm{P}$, or NEXPTIME, depending on the input.

In particular, for homogeneous PDS over a maxterm (or minterm) Boolean function, a crucial result in [33] proves that if $F$ is a MAX - PDS (or MIN - PDS) over a dependency graph $G$, then all the periodic points of this system are fixed points, or all of them are 2-periodic points. Actually, the authors found necessary and sufficient conditions under which all the periodic points of a MAX - PDS (resp., a MIN - PDS) are fixed points or all of them are 2-periodic points. When all the periodic points of the system are fixed points, we simply say that $F$ is a fixed point system and when all of them are 2-periodic points, we say $F$ is a 2 -periodic point system. In addition, a criterion was given to check if the system has a unique fixed point.

Later, in [34], the authors found a characterization of MAX - PDS (resp., a MIN - PDS) to have a unique 2-periodic orbit. Moreover, an upper bound for the number of fixed points and the number of 2-periodic points of such systems were also obtained in [34], thus solving the maximum number problems in this context.

In this work, our main goal is to provide lower bounds for the number of fixed points and the number of periodic points of an arbitrary MAX - PDS (and MIN - PDS), i.e., to solve the minimum number problems. Besides, we provide formulae for counting (exactly) such points for PDS over different particular classes of graphs. The results are shown here only for MAX - PDS, since they can be easily translated to the case of MIN - PDS by duality. Likewise, the results on fixed points are also valid for MAX-SDS (and MIN-SDS) since, as well known, fixed points of an SDS are the same as the corresponding PDS.

This paper is organized as follows. Section 2 is devoted to counting fixed points and establishing the minimum number of them. For an arbitrary PDS on a maxterm, namely, $F$, over a dependency graph $G=(V, E)$, we induce a bipartite graph $B_{F}$ on a vertex set $V_{1} \cup \dot{U} V_{2}$ (see Definition 2) and define the notion of dominating sets of $V_{2}$ in $B_{F}$ (see Definition 1). Next, we provide a characterization of the fixed points of $F$ in terms of such dominating sets (see Theorem 2). The bijective correspondence between the fixed points and dominating sets allows us to reformulate the known theorems on the existence and uniqueness of fixed points in terms of such dominating sets (see Theorem 3). Such a correspondence also allows us to get the same upper bound for number of fixed points as in [34]. Moreover, the new method to obtain this upper bound provides a characterization to attain this maximum number of fixed points (see Corollary 1). Finally, we consider minimal dominating sets and provide a lower (combinatorial) bound for the number of fixed points of $F$ (see Theorem 4). Furthermore, we are able to get a characterization for MAX - PDS to have such minimum number of fixed points in Theorem 5 , thus solving the minimum number of fixed-point problems for this kind of PDS. Note that Theorem 5 can be considered as a generalization of the fixed-point theorem ([33], Theorem 9). In this section, we also provide a formula to easily count (exactly) the fixed points (see Theorem 6). In Section 3, we assume that $F$ is a 2-periodic point MAX - PDS and study the number of its 2-periodic points. In this case, we use a counting method based on the notion of maximal independent sets of a graph and the corresponding independence number. First, we provide a lower bound for the number of 2-periodic points in the case of the maxterm NAND (see Theorem 7). Moreover, in such a case, we provide a sufficient condition to attain this lower bound in Corollary 3 . As a consequence, we give a simple formula for counting (exactly) the 2 -periodic points when $G$ is a $t$-partite graph or a $(n-2)$ regular graph (see Corollary 3$)$. Finally, by combining the previous results, we give a lower combinatorial bound for the number of 2-periodic points of a MAX - PDS (see Theorem 8). We also provide an example to prove that this bound is the best possible one, i.e., it is the minimum number of 2-periodic points. We conclude the paper giving some conclusions and future research directions in Section 4.

\section{Minimum Number of Fixed Points in PDS}

First, we recall a crucial theorem for characterizing MAX - PDS which are fixed-point systems and for determining when a MAX - PDS has a unique fixed point. 
Theorem 1 (see [33], Theorems 3 and 9). Let $F$ be a MAX $P D S$ over a dependency graph $G=(V, E), G_{1}, \ldots, G_{p}$ be the connected components of $G \backslash W^{\prime}$, and

$$
W_{1}^{\prime}=\left\{i \in W^{\prime}: \exists ! 1 \leq j \leq p \text {, such that } i \in A_{G}\left(V\left(G_{j}\right)\right)\right\} \text {, }
$$

where $V\left(G_{j}\right)$ is the set of vertices of $G_{j}$. Then,

(1) $F$ is a fixed point system if, and only if, $W \cap A_{G}(i) \neq \varnothing$ for all $i \in W^{\prime}$.

(2) If $F$ is a fixed point system, then $F$ has a unique fixed point if and only if for all $1 \leq j \leq p$, $A_{G}\left(V\left(G_{j}\right)\right) \cap W_{1}^{\prime} \neq \varnothing$. In such a case, the unique fixed point of the system is the one with all the state values equal to 1 .

Remark 1 (see [33], Section 3). We also know that if $\hat{x}=$ $\left(x_{1}, \ldots, x_{n}\right)$ is a fixed point, then

(fp1) All the variables associated with the vertices in $W^{\prime}$ are activated

(fp2) For each $1 \leq i \leq p$, all the variables associated with the vertices in $G_{i}$ are activated, or all of them are deactivated

(fp3) Moreover, if $G_{j_{1}}, G_{j_{2}}, \ldots, G_{j_{l}}$ are the connected components adjacent to a vertex $j \in W^{\prime}$, then not all (the variables associated with the vertices in) these components can be deactivated simultaneously; otherwise, the value of $x_{j}$ would change from 1 to 0 in the following iteration.

From this characterization of fixed points, in Theorem 1 of [34], it was stated that the number of fixed points of a fixed-point system MAX - PDS over a connected graph with MAX $\neq \mathrm{OR}$ is upper bounded by $2^{p}-1$. For the well-known case, when $\mathrm{MAX}=\mathrm{OR}$, the number of fixed points is equal to 2.

Taking these results into account, we then provide a novel combinatorial characterization of the fixed points of a MAX - PDS. In order to do this, we need some notions of graph theory.

Definition 1. Let $B$ be a bipartite graph whose disjoint sets of vertices are $V_{1}$ and $V_{2}$ (i.e., $V_{1} \dot{\cup} V_{2}=V(B)$ and each edge of $B$ has an endpoint in $V_{1}$ and an endpoint in $\left.V_{2}\right)$. Then, we say that $S \subseteq V_{1}$ is a dominating set of $V_{2}$ in $B$ if every vertex in $V_{2}$ is adjacent to at least one in $S$. In this situation, we define

$$
\eta(B)=\min \left\{|S|: S \subseteq V_{1} \text { is a dominating set of } V_{2} \text { in } B\right\} .
$$

Note that Definition 1 is an adaptation of the usual notion of dominating set in graph theory to bipartite graphs.

Next, for each MAX - PDS over a dependency graph $G$, we induce a bipartite graph $B$.

Definition 2. Let $F$ be a MAX - PDS over a dependency graph $G=(V, E)$ with $\varnothing \neq W^{\prime} \subsetneq V$, and let $G_{1}, \ldots, G_{p}$ be the connected components of $G \backslash W^{\prime}$. Associated with this
MAX - PDS, we define a bipartite graph $B_{F}$ in which $V\left(B_{F}\right)=V_{1} \dot{U} V_{2}$ as

(i) $V_{1}=\{\overline{1}, \ldots, \bar{p}\}$. Intuitively, we collapse the connected component $G_{i}$ into a single vertex $\bar{i}$ for $i=1, \ldots, p$.

(ii) $V_{2}=W^{\prime}$.

(iii) The edge set of $B_{F}$ is defined as

$$
\{\bar{i}, j\} \in E\left(B_{F}\right) \Longleftrightarrow \exists k \in G_{i} \text { adjacent to } j \text {. }
$$

Example 1. Assume that $G=(V, E)$ is the graph of Figure 1 . Let us take

$$
\begin{aligned}
& f\left(x_{1}, x_{2}, x_{3}, x_{4}, x_{5}, x_{6}, x_{7}, x_{8}, x_{9}\right) \\
& \quad=x_{1} \vee x_{2} \vee x_{3} \vee x_{4} \vee x_{5} \vee x_{6} \vee x_{7}^{\prime} \vee x_{8}^{\prime} \vee x_{9}^{\prime} .
\end{aligned}
$$

Then, $W^{\prime}=\{7,8,9\}$ and $G \backslash W^{\prime}$ has 4 connected components: $G_{1}=\{1,2\}$, which we collapse into the vertex $\overline{1}$; $G_{2}=\{3\}$, which we collapse into the vertex $\overline{2} ; G_{3}=\{4\}$, which we collapse into the vertex $\overline{3}$; and $G_{4}=\{5,6\}$, which we collapse into the vertex $\overline{4}$. Then, bearing in mind the edges between the vertices in $W^{\prime}$ and those in the components $G_{i}$, the bipartite graph $B_{F}$ becomes as shown in Figure 2:

As a key point for our study, we then show that there is a bijective correspondence between the dominating sets of $V_{2}$ in $B_{F}$ and the fixed points in the MAX - PDS system.

Theorem 2. Let $F$ be a MAX - PDS system over a dependency graph $G=(V, E)$ with $W^{\prime} \neq \varnothing, G_{1}, \ldots, G_{p}$ be the connected components of $G \backslash W^{\prime}$ and $B_{F}$ be the associated bipartite graph. Then, there exists a bijective correspondence between the dominating sets of $V_{2}$ in $B_{F}$ and the fixed points of the system.

In particular

$$
|\operatorname{Fix}(F)|=\mid\left\{S \subseteq V_{1} \text { : Sis a dominating set of } V_{2} \text { in } B_{F}\right\} \mid \text {, }
$$

where $\operatorname{Fix}(F)$ is the set of fixed points of the system.

Proof. Let $\Sigma$ be the set of dominating sets of $V_{2}$ in $B_{F}$ and let us consider the map $\Psi: \Sigma \longrightarrow \operatorname{Fix}(F)$ defined as follows: given $S \in \Sigma$, we take $\Psi(S)$ as the point $x=\left(x_{1}, \ldots, x_{n}\right) \in\{0,1\}^{n}$ such that

$\left(\Psi_{1}\right)$ All the variables associated with the vertices in $W^{\prime}$ are activated

$\left(\Psi_{2}\right)$ For each $i, 1 \leq i \leq p$, if $\bar{i} \in S$, then all the variables associated with the vertices in $G_{i}$ are activated; otherwise, all the variables associated with the vertices in $G_{i}$ are deactivated

Observe that $\Psi(S)$ is a fixed point of the system (see Remark 1), i.e., $\Psi$ is well-defined. Moreover $\Psi$ is injective since $\Psi\left(S_{1}\right) \neq \Psi\left(S_{2}\right)$ for $S_{1}, S_{2} \in \Sigma$ with $S_{1} \neq S_{2}$. Finally, given 


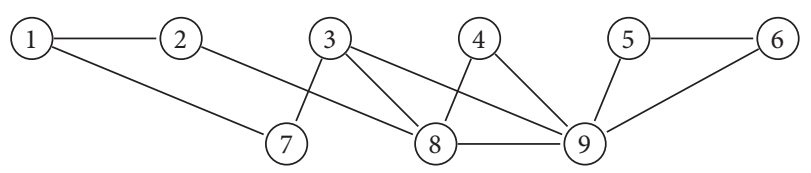

Figure 1: Graph G.

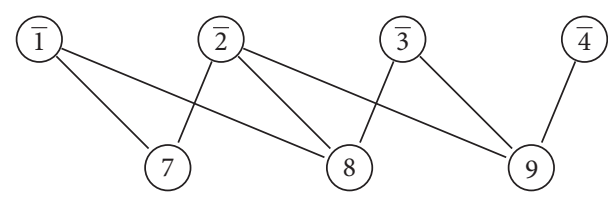

Figure 2: Graph $B_{F}$.

a fixed point $x \in \operatorname{Fix}(F)$ and taking into account (fp1), (fp2), and (fp3) in Remark 1, the subset $S$ of $V_{1}$, whose elements are the vertices $\bar{i}$ such that the vertices of $G_{i}$ are activated in $x$, is a dominating set of $V_{2}$ verifying that $\Psi(S)=x$, i.e., $\Psi$ is surjective.

With all of these, we can rewrite Theorem 1 to check if a MAX - PDS is a fixed-point system and if it has a unique fixed point in terms of the bipartite graph $B_{F}$.

Theorem 3. Let $F$ be a MAX - PDS system over a dependency graph $G=(V, E)$ with $\varnothing \neq W^{\prime} \subsetneq V$, and $B_{F}$, the associated bipartite graph. Then,

(i) $F$ is a fixed point system if, and only if, $V_{1}$ is a dominating set of $V_{2}$ in $B_{F}$

(ii) $F$ has a unique fixed point if, and only if, $V_{1}$ is the unique dominating set of $V_{2}$ in $B_{F}$

Proof

(i) By Theorem 1, $F$ is a fixed point system if and only if $W \cap A_{G}(j) \neq \varnothing$ for all $j \in W^{\prime}$. But this occurs, if and only if, for all $j \in W^{\prime}$, there exists $i, 1 \leq i \leq p$ such that $j \in A_{G}\left(V\left(G_{i}\right)\right)$, which is equivalent to say that $V_{1}$ is a dominating set of $V_{2}$ in $B_{F}$. Therefore, the conclusion follows.

(ii) By Theorem 2, every fixed point corresponds to a dominating set. Thus, we have a unique fixed point if, and only if, we have a unique dominating set in $G$. This occurs if, and only if, $V_{1}$ is the unique dominating set.

Let $F$ be a MAX - PDS over a dependency graph $G=$ $(V, E)$ in which $\varnothing \neq W^{\prime} \subsetneq V$. In Theorem 1 of [34], it is proved that the maximum number of fixed points for this system is $2^{p}-1$, where $p$ is the number of connected components of $G \backslash W^{\prime}$. In the next corollary, we find a necessary and sufficient condition for achieving this upper bound. Recall that a bipartite graph with $V=V_{1} \dot{U} V_{2}$ is said to be a complete bipartite graph, if each node of $V_{1}$ is adjacent to all the nodes in $V_{2}$.
Corollary 1. Let $F$ be a MAX - PDS system over a dependency graph $G=(V, E)$ with $\varnothing \neq W^{\prime} \subsetneq V, G_{1}, \ldots, G_{p}$ be the connected components of $G \backslash W^{\prime}$, and $B_{F}$ be the associated bipartite graph. Then, $\mid$ Fix $(F) \mid=2^{p}-1$ if, and only if, $B_{F}$ is a complete bipartite graph.

Proof. It is enough to note that, by Theorem 2, $|\operatorname{Fix}(F)|$ is maximum if, and only if, each arbitrary nonempty subset of $V_{1}$ is a dominating set of $V_{2}$ in $B_{F}$ which is equivalent to say that $B_{F}$ is a complete bipartite graph.

Let us consider the order relation on $\Sigma$ defined by

$$
S_{1} \leq S_{2} \Longleftrightarrow S_{1} \subseteq S_{2}, \quad S_{1}, S_{2} \in \Sigma .
$$

Regarding this order, a dominating set $S_{1}$ is said to be minimal if there is not another dominating set $S_{2}$ such that $S_{2} \nsubseteq S_{1}$.

Note that if $S$ a dominating set of $V_{2}$ and $T \subseteq V_{1}$ is such that $S \subseteq T$, then $T$ is also a dominating set.

With all of these, we can provide a lower bound for the number of fixed points in a PDS as follows.

Theorem 4. Let $F$ be a MAX-PDS system over a dependency graph $G=(V, E)$ with $\varnothing \neq W^{\prime} \subsetneq V, G_{1}, \ldots, G_{p}$ be the connected components of $G \backslash W^{\prime}$, and $B_{F}$ be the associated bipartite graph. Then, the number of fixed points is, at least, $2^{p-\eta\left(B_{F}\right)}$. That is,

$$
|\operatorname{Fix}(F)| \geq 2^{p-\eta\left(B_{F}\right)} .
$$

Proof Given $S$ a dominating set such that $|S|=\eta\left(B_{F}\right)$, we know that $S \cup T$ is also a dominating set of $V_{2}$ for each $T \subseteq V_{1} \backslash S$.

Since $\left|V_{1} \backslash S\right|=p-\eta\left(B_{F}\right)$, the possibilities of choosing $T \subseteq V_{1} \backslash S$ are $2^{p-\eta\left(B_{F}\right)}$. Therefore, we have at least such number of dominating sets and, as a consequence of Theorem 2, at least such number of fixed points.

Once we know the lower bound for the number of fixed points, we can establish a minimum number of fixed-point theorem, thus generalizing the fixed-point theorem given for PDS in [33], as follows.

Theorem 5 (minimum number of fixed-point theorem). Let $F$ be a MAX - PDS system over a dependency graph $G=$ $(V, E)$ with $\varnothing \neq W^{\prime} \subsetneq V, G_{1}, \ldots, G_{p}$ be the connected components of $G \backslash W^{\prime}$, and $B_{F}$ be the associated bipartite graph. Then, the number of fixed points is minimum if, and only if, there exists a unique minimal dominating set of $V_{2}$ in $B_{F}$. In such a case, this minimum number of fixed points is given by

$$
|\operatorname{Fix}(F)|=2^{p-\eta\left(B_{F}\right)} .
$$

Proof. Observe that the proof consists in getting the equality in the formula of Theorem 4 under the assumption of the existence of a unique minimal dominating set. Certainly, the equality follows under this assumption by taking into 
account that, if $S$ is the unique minimal dominating set of $V_{2}$, then $|S|=\eta\left(B_{F}\right)$ and every other dominating set of $V_{2}$ is of the form $S \cup T$ for $T \subseteq V_{1} S$.

Conversely, if $S_{1}$ and $S_{2}$ are two different minimal dominating sets of $V_{2}$ in $B_{F}$ and $\left|S_{1}\right|=\eta\left(B_{F}\right)$, then $S_{2} \notin\left\{S_{1} \cup T ; T \subseteq V_{1} \backslash S_{1}\right\}$. Thus, $F$ would have at least $2^{p-\eta\left(B_{F}\right)}+1$ fixed points.

Example 2. Let $G$ be the graph of Figure 3.

Assume that

$f\left(x_{1}, x_{2}, x_{3}, x_{4}, x_{5}, x_{6}, x_{7}\right)=x_{1} \vee x_{2} \vee x_{3} \vee x_{4} \vee x_{5} \vee x_{6}^{\prime} \vee x_{7}^{\prime}$.

Then, by definition, $B_{F}$ is the graph of Figure 4 .

It is easy to see that $\{\overline{1}\}$ is the unique minimal dominating set of $\{6,7\}$ in $B_{F}, \eta\left(B_{F}\right)=1$, and $G \backslash W^{\prime}$ has 3 connected components. By Theorem $4, F$ has 4 fixed points which are (see Theorem 3)

$$
\begin{aligned}
\operatorname{Fix}(F)= & \{(1,1,1,1,1,1,1),(1,1,1,1,0,1,1), \\
& (1,1,1,0,0,1,1),(1,1,1,0,1,1,1)\} .
\end{aligned}
$$
and

Let us take $M$ the set of minimal dominating sets of $V_{2}$

$$
\bar{S}=\underset{S \in M}{\cup} S,
$$

and then the union of all the minimal dominating sets of $V_{2}$.

As stated in Theorem 4, if $|M|=1$, we have that

$$
|\operatorname{Fix}(F)|=2^{p-\eta\left(B_{F}\right)} .
$$

In this line, we are able to provide a formula for counting (exactly) the fixed points of the system.

Theorem 6. Let $F$ be a MAX - PDS system over a dependency graph $G=(V, E)$ with $\varnothing \neq W^{\prime} \subsetneq V, G_{1}, \ldots, G_{p}$ be the connected components of $G \backslash W^{\prime}$, and $B_{F}$ be the associated bipartite graph. Let $M=\left\{S_{1}, \ldots, S_{q}\right\}$ be the set of minimal dominating sets of $V_{2}$ in $B_{F}, \bar{S}=\cup_{j=1}^{q_{1}} S_{j}$, and

$$
\Omega=\left\{S \subseteq V_{1}: S_{j} \subseteq S \subseteq \bar{S} \text { for some } j 1 \leq j \leq q\right\} .
$$

Then,

$$
|\operatorname{Fix}(F)|=|\Omega| 2^{p-|\bar{S}|}
$$

Proof. The equality follows from Theorem 3 taking into account that if $S$ is a dominating set of $V_{2}, S \cup T$ is also a dominating set of $V_{2}$ for all $T \subseteq V_{1} \backslash S$.

As a consequence, we have the following result when $q \geq 2$ :

Corollary 2. Let $F$ be a MAX - PDS system over a dependency graph $G=(V, E)$ with $\varnothing \neq W^{\prime} \subsetneq V, G_{1}, \ldots, G_{p}$ be the connected components of $G W^{\prime}$, and $B_{F}$ be the associated bipartite graph. Let $M=\left\{S_{1}, \ldots, S_{q}\right\} \quad(q \geq 2)$ be the set of

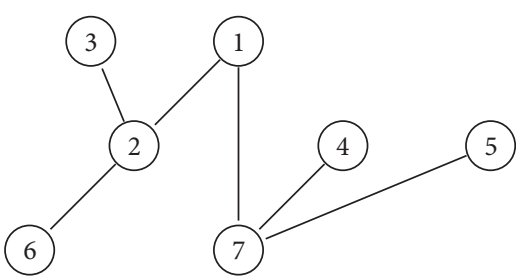

FIGURE 3: Graph G.

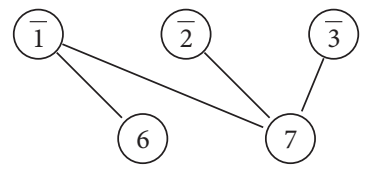

Figure 4: Graph $B_{F}$.

minimal dominating sets of $V_{2}$ in $B_{F}, \bar{S}=\cup_{j=1}^{q} S_{j}$, and assume that $\left|\bar{S} \backslash S_{j}\right|=1$ for all $j, 1 \leq j \leq q$. Then,

$$
|\operatorname{Fix}(F)|=(q+1) 2^{p-|\bar{S}|} .
$$

Remark 2. Let $F$ be an arbitrary MIN-PDS over a dependency graph $G=(V, E)$. Then, all the results of this section can be restated for $F$ by duality.

\section{Minimum Number of 2-Periodic Points in PDS}

In this section, we provide a lower bound for the number of 2-periodic points of a MAX - PDS when it is a 2-periodic point system. To do this, we will use Proposition 3 in [34], where the number of 2-periodic orbits of a NAND-PDS is computed:

Proposition 1 (see [34], Proposition 3). Let $F$ be the $N A N D-P D S$ over the dependency graph $G=(V, E)$ and $\mathscr{P}(V)$ be the power set of $V$. Then, the number of 2-periodic points of this system is $|\Theta|$, where

$$
\Theta=\left\{\overline{A_{G}(Q)}: Q \in \mathscr{P}(V)\right\} .
$$

Specifically, in this proposition, it is proved that $x=\left(x_{1}, \ldots, x_{n}\right) \in \operatorname{Per}_{2}(F), \quad$ if and only if $\left\{j: x_{j}=1\right\} \in \Theta$,

where $\operatorname{Per}_{2}(F)$ is the set of 2-periodic points of the system.

Given $G=(V, E)$, we say that $S \subset V$ is an independent set of $G$ if $\{i, j\} \notin E$ for whichever $i, j \in S$. Furthermore, $S$ is said to be a maximal independent set if $S \cup\{k\}$ is not independent for all $k \in V \backslash S$. The independence number of $G$, $\alpha(G)$, is then defined as

$$
\alpha(G)=\max \{|S|: S \subseteq V(G) \text { is an independent set }\} .
$$

In the next result, we give a lower bound for the number of 2-periodic points of a NAND - PDS. 


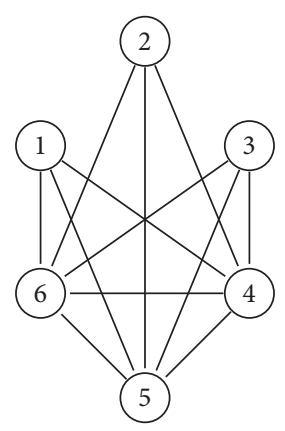

Figure 5: Graph G.

Theorem 7. Let $F$ be the NAND-PDS over a dependency graph $G=(V, E)$. Then,

$$
\left|\operatorname{Per}_{2}(F)\right| \geq 2^{\alpha(G)} .
$$

Proof. Given $F$ a NAND-PDS over $G$, we know by Proposition 1 that $\left|\operatorname{Per}_{2}(F)\right|=|\Theta|$.

Let $S$ be a maximal independent set of $G$ with $|S|=\alpha(G)$. One can easily see that for any two distinct subsets $Q_{1}$ and $Q_{2}$ of $S, \overline{A_{G}\left(Q_{1}\right)} \neq \overline{A_{G}\left(Q_{2}\right)}$. Therefore, $\Theta$ has, at least, $2^{\alpha(G)}$ elements.

As a consequence, we have the following.

Corollary 3. Let $F$ be the NAND - PDS over the dependency graph $G=(V, E)$, and let $S$ be a maximal independent set of $G$ such that $|S|=\alpha(G)$ and $\overline{A_{G}(i)}=V$ for each $i \in V \backslash S$. Then, $|\Theta|=2^{\alpha(G)}$.

Proof. As shown in the proof of Theorem 7, for every two different subsets of $S$, we have two different 2-periodic points.

On the other hand, for any $Q \not \subset S$, there is $i \in Q$ such that $i \in V \backslash S$. Hence, $V=A_{G}(i) \subset A_{G}(Q)$. As $S$ is a maximal independent set, $A_{G}(S)=V=A_{G}(Q)$. Thus, only distinct subsets of $S$ generate different periodic points, and the equality holds.

In particular, if $G$ is a complete graph, then it satisfies the conditions of the Corollary, being $\alpha(G)=1$, and the number of periodic points of the corresponding NAND - PDS coincides with $2^{\alpha(G)}=2$ ([34], Proposition 1), i.e., the bound provided in Theorem 7 is attained.

This situation in Corollary 3 also happens, for example, when $G$ is a star graph where the center is the unique point outside the maximal independent set. Thus, the number of 2periodic points is $2^{n-1}$, which matches with the lower bound (see [34], Example 2).

Let us present another example.

Example 3. Let $G$ be the graph of Figure 5 .

One can easily see that $\{1,2,3\}$ is a maximal independent set in $G$ and $\alpha(G)=3$. Moreover, $\overline{A_{G}(4)}=$ $\overline{A_{G}(5)}=\overline{A_{G}(6)}=\{1,2,3,4,5,6\}$. Then, from Corollary 3, we have that $|\Theta|=2^{3}=8$. Actually, $\Theta=\left\{\overline{A_{G}(Q)}: Q \subseteq\{1,2,3\}\right.$.
Next, we compute the number of 2-periodic points of a NAND - PDS over a complete $t$-partite graph. Recall that the complete $t$-partite graph $G=K_{n_{1}, \ldots, n_{t}}$ is a graph whose set of nodes $V$ is the union of pairwise disjoint subsets $V_{1}, \ldots, V_{t}$, with $\left|V_{i}\right|=n_{i}$, and such that each vertex in $V_{i}$ is adjacent to all the vertices in $V \backslash V_{i}, i=1, \ldots, t$.

Corollary 4. Let $F$ be the NAND-PDS over the complete t-partite graph $G=K_{n_{1}, \ldots, n_{t}}$. Then,

$$
\left|\operatorname{Per}_{2}(F)\right|=\sum_{i=1}^{t} 2^{n_{i}}-2(t-1) .
$$

In particular, if $G$ is the $(n-2)$-regular graph, then $\left|\operatorname{Per}_{2}(F)\right|=n+2$.

Proof. Let $G=K_{n_{1}, \ldots, n_{t}}$ be the complete $t$-partite graph on the vertex set $V=V_{1} \dot{U} \cdots \dot{U} V_{t}$. For any $\varnothing \neq Q \subseteq V$, two situations are possible:

(i) If $Q \subseteq V_{i}$ for some $1 \leq i \leq t$, then $\overline{A_{G}(Q)}=Q \cup\left(V \backslash V_{i}\right)$

(ii) If $Q$ is not contained in $V_{i}$ for any $1 \leq i \leq t$, then $\overline{A_{G}(Q)}=V$ that

Taking also into account that $\overline{A_{G}(\varnothing)}=\varnothing$, we conclude

$$
\Theta=\{V, \varnothing\} \cup\left\{\overline{A_{G}(Q)}: \varnothing \neq Q \subsetneq V_{i}, 1 \leq i \leq t\right\},
$$

and so

$$
\left|\operatorname{Per}_{2}(F)\right|=|\Theta|=2+\sum_{i=1}^{t} 2^{n_{i}}-2 t .
$$

It is easy to check that if $G$ is $(n-2)$-regular, then $n$ is even and $G$ is a complete $n / 2$-partite graph in which every $V_{i}$ has exactly 2elements (specifically each $V_{i}$ consists in a pair of nonadjacent vertices). Therefore,

$$
\left|\operatorname{Per}_{2}(F)\right|=2+\frac{n}{2} 2^{2}-2 \frac{n}{2}=n+2 .
$$

Given an arbitrary 2-periodic point system MAX - PDS over a dependency graph $G=(V, E)$, we are able to obtain a lower bound for $\left|\operatorname{Per}_{2}(F)\right|$ by using Theorems 4 and 7 . We will assume that both $W$ and $W^{\prime}$ are nonempty sets since the particular cases $W=\varnothing$ and $W^{\prime}=\varnothing$ have been already analyzed in detail.

Consider $W^{\prime}$ as the disjoint union of $W_{D}^{\prime}$ and $W_{C}^{\prime}$, where

$$
\begin{aligned}
& W_{D}^{\prime}=\left\{i \in W^{\prime}: A_{G}(i) \cap W \neq \varnothing\right\}, \\
& W_{C}^{\prime}=\left\{i \in W^{\prime}: A_{G}(i) \cap W=\varnothing\right\} .
\end{aligned}
$$

The restriction of the system to the vertex set $W \cup W_{D}^{\prime}$, $\left.F\right|_{W \cup W_{D}^{\prime}}$, is a fixed-point MAX - PDS over the induced subgraph $G_{D}$ of $G$ whose vertex set is $W \cup W_{D}^{\prime}$ (see [34], Section 3). We also know that the restriction of $F$ to $W_{C}^{\prime}$, $\left.F\right|_{W_{C}^{\prime}}$, is a 2-periodic point system over the induced subgraph $G_{C}$ of $G$ whose vertex set is $W_{C}^{\prime}$. 
Let $x=\left(x_{1}, \ldots, x_{n}\right)$ be an arbitrary point of $\{0,1\}^{n}$. Without loss of generality, let us assume that $x_{1}, \ldots, x_{m}$ are the variables associated with the vertices in $W \cup W_{D}^{\prime}$ and $x_{m+1}, \ldots, x_{n}$ those associated with the vertices in $W_{C}^{\prime}$.

Observe that if $\left(x_{1}, \ldots, x_{m}\right)$ is a fixed point of $\left.F\right|_{W \cup W_{D}^{\prime}}$ and $\left(x_{m+1}, \ldots, x_{n}\right)$ is a 2 -periodic point of $\left.F\right|_{W_{c}^{\prime}}$, then $x$ is a 2 -periodic point of $F$. In this situation, the variables associated with the vertices in $W_{D}^{\prime}$ are fixed to 1 , and so they do not affect the updating of the variables associated with vertices in $W_{C}^{\prime}$ anymore (see [34], Section 3, for more details).

Therefore,

$$
\left|\operatorname{Per}_{2}(F)\right| \geq\left|\operatorname{Per}_{2}\left(\left.F\right|_{W_{C}^{\prime}}\right)\right| \times\left|\operatorname{Fix}\left(\left.F\right|_{W \cup W_{D}^{\prime}}\right)\right| .
$$

In fact, inequality (27) may be strict, as shown in the following example.

Example 4. Let $G$ be the graph of Figure 6 .

Assume that $F$ is the homogeneous MAX - PDS over G whose evolution operator is demaxterm

$$
f\left(x_{1}, x_{2}, x_{3}, x_{4}, x_{5}\right)=x_{1} \vee x_{2} \vee x_{3}^{\prime} \vee x_{4}^{\prime} \vee x_{5}^{\prime} \text {. }
$$

Then, it can be easily checked that $x=(0,0,1,1,0)$ is a 2 -periodic point of $F(F(x)=(0,0,0,1,1))$, but $(0,0,1)$, associated with the vertices 1,2 , and 4 , is not a fixed point of $\left.F\right|_{G_{D}}\left(\left.F\right|_{G_{D}}(0,0,1)=(0,0,0)\right)$. Hence, for this system, inequality (27) is strict.

By combining Theorems 4 and 7, we have the following result:

Theorem 8. Let $F$ be an arbitrary $M A X-P D S$ over a dependency graph $G$ with $\varnothing \neq W^{\prime} \subsetneq V$. If $F$ is a 2-periodic point system, then

$$
\left|\operatorname{Per}_{2}(F)\right| \geq 2^{p-\eta\left(\left.B_{F \mid}\right|_{G_{D}}\right)} 2^{\alpha\left(G_{C}\right)},
$$

where $p$ is the number of connected components of $G \backslash W^{\prime}$.

We next give an example in which the number of 2 -periodic points is exactly the same as the lower bound in Theorem 8.

Example 5. Consider the graph $G$ of Figure 7.

If we define $f$ as

$$
f\left(x_{1}, x_{2}, x_{3}, x_{4}, x_{5}\right)=x_{1} \vee x_{2} \vee x_{3}^{\prime} \vee x_{4}^{\prime} \vee x_{5}^{\prime},
$$

and $F$ is the corresponding homogeneous MAX - PDS, then we have

$$
\begin{aligned}
W & =\{1,2\}, \\
W_{D}^{\prime} & =\{3,4\}, \\
W_{C}^{\prime} & =\{5\} .
\end{aligned}
$$

The two connected components of $G \backslash W^{\prime}=G \backslash$ $\left(W_{D}^{\prime} \cup W_{C}^{\prime}\right)$ consist of the isolated vertices 1 and 2, respectively. Let $G_{D}$ be the induced subgraph of $G$ on the

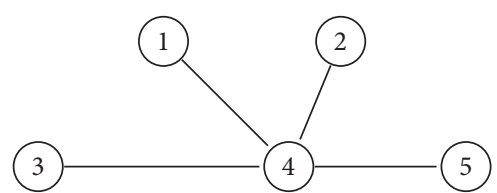

Figure 6: Graph G.

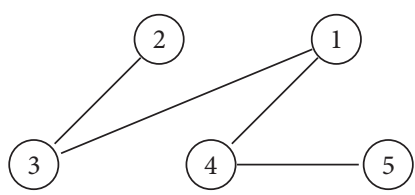

Figure 7: Graph G.

vertex set $W \cup W_{D}^{\prime}=\{1,2\} \cup\{3,4\}$. For the corresponding bipartite graph $B_{F_{G_{D}}}$, we have

$$
\begin{aligned}
V\left(B_{\left.F\right|_{G_{D}}}\right) & =\{\overline{1}, \overline{2}\} \dot{\cup}\{3,4\}, \\
E\left(B_{\left.F\right|_{G_{D}}}\right) & =\{\{\overline{1}, 3\},\{\overline{1}, 4\}\} \cup\{\{\overline{2}, 3\}\} .
\end{aligned}
$$

It is clear that $\{\overline{1}\}$ is the unique minimal dominating set of $V_{2}=\{3,4\}$ in $B_{\left.F\right|_{G_{D}}}$. Thus, by Theorem 3, a point $x=$ $\left(x_{1}, x_{2}, x_{3}, x_{4}, x_{5}\right)$ is a fixed point of $\left.F\right|_{G_{D}}$ if and only if $x_{1}=x_{3}=x_{4}=1$, and the number of fixed points of $\left.F\right|_{G_{D}}$ is $2^{2-1}$. Finally, note that $G_{C}$, the induced subgraph on the vertex set $W_{C}^{\prime}$, is an isolated vertex. So, $\operatorname{Per}_{2}\left(\left.F\right|_{G_{C}}\right)=$ $\{(1),(0)\}$ and $\left|\operatorname{Per}_{2}\left(F||_{G_{C}}\right)\right|=2^{\alpha\left(G_{C}\right)}=2$.

In the following, for each $j \geq 0$, we denote by $\left(x_{1}^{j}, \ldots, x_{5}^{j}\right)$ the point $F^{j}(x)$ where $x=\left(x_{1}^{0}, \ldots, x_{5}^{0}\right)$.

Assume that $x=\left(x_{1}^{0}, x_{2}^{0}, x_{3}^{0}, x_{4}^{0}, x_{5}^{0}\right) \in \operatorname{Per}_{2}(F)$. Then, by the same discussion before ([34], Theorem 5), $x_{3}^{0}=x_{4}^{0}=1$ (so, for each $j \geq 0, x_{3}^{j}=x_{4}^{j}=1$ ) and $x_{5}^{0}=0$ or 1 .

We shall show that $\left(x_{1}^{0}, x_{2}^{0}, x_{3}^{0}, x_{4}^{0}\right)$ is a fixed point of $\left.F\right|_{G_{D}}$. Actually, if it is not the case, then $x_{1}^{0}=0$ and so, $x=\left(0, x_{2}^{0}, 1,1,1\right)$ or $x=\left(0, x_{2}^{0}, 1,1,0\right)$. Hence, looking to $f_{4}=x_{1} \vee x_{4}^{\prime} \vee x_{5}^{\prime}$ we have that $x_{4}^{1}=0$ or $x_{4}^{2}=0$ which is a contradiction. Thus, $\left(x_{1}^{0}, x_{2}^{0}, x_{3}^{0}, x_{4}^{0}\right)$ is a fixed point of $\left.F\right|_{G_{D}}$.

Therefore, a point $x=\left(x_{1}, x_{2}, x_{3}, x_{4}, x_{5}\right)$ is a 2 -periodic point of $F$ if, and only if, $\left(x_{1}, x_{2}, x_{3}, x_{4}\right)$ is a fixed point of $\left.F\right|_{G_{D}}$ and $\left(x_{5}\right)$ is a 2-periodic point of $\left.F\right|_{G_{C}}$. In particular,

$$
\begin{aligned}
\left|\operatorname{Per}_{2}(F)\right|= & \left|\operatorname{Fix}\left(\left.F\right|_{G_{D}}\right)\right| \times\left|\operatorname{Per}_{2}\left(\left.F\right|_{G_{C}}\right)\right|=2^{m-\eta\left(D_{F \mid G_{D}}\right)} 2^{\alpha\left(G_{C}\right)} \\
& =2^{2-1} \times 2=2^{2} .
\end{aligned}
$$

Specifically, in our case, $\operatorname{Per}_{2}(F)=\{(1,0,1,1,1)$, $(1,0,1,1,0),(1,1,1,1,1),(1,1,1,1,0)\}$.

Remark 3. Let $F$ be an arbitrary MIN - PDS over a dependency graph $G=(V, E)$. Then, all the results of this section can restated for $F$ by duality. Moreover, since the fixed points of a PDS coincide with the ones of any SDS with the same dependency graph and local functions, whichever the update order is, the results obtained in this section are also valid for the case of sequential dynamical systems of the form MAX-SDS and MIN-SDS. 


\section{Conclusions and Future Research Directions}

In this paper, we have shown that the problem of counting the fixed points of a homogeneous MAX-PDS can be transformed into the problem of counting minimal dominating sets of an ad hoc bipartite graph constructed from the dependency graph of the system. The method used here has two advantages: first, we can get the results in previous works on the number of fixed points by means of more simple conditions in terms of minimal dominating sets; second, using this new approach, we have been able to provide a lower bound for the number of fixed points of an arbitrary MAX - PDS and to generalize the fixed-point theorem stated in [33] with a minimum number of fixed point theorem; even more, we have also provided a formula to calculate the exact number of such fixed points. Of course, the results are also applicable to any MIN-PDS by duality. Moreover, since the fixed points of a PDS coincide with the ones of any SDS with the same dependency graph and local functions, whichever the update order is, the results on fixed points obtained are also valid for the case of sequential dynamical systems of the form MAX-SDS and MIN-SDS.

In addition, we have got a lower bound for the number of 2-periodic points of an arbitrary NAND - PDS and, in general, of any MAX - PDS, in terms of the independence number of the dependency graph. Since the independence number is known for many classes of graphs (e.g., complete, path, cycle, and complete $k$-partite graphs), this method can be useful in many cases. By duality, such lower bounds are also valid to any NOR-PDS and any MIN-PDS, respectively.

Our results open some future research directions to be discovered. Actually, we think that, using ideas like the ones in this paper, periodic points of MAX-PDS (resp., MIN - PDS) over directed graphs [27] could be better analyzed. The techniques employed here could be also interesting to extend this study to sequential dynamical systems, i.e., to those systems where the entities are updated asynchronously.

\section{Data Availability}

The data used to support the findings of this study are included within the article.

\section{Conflicts of Interest}

The authors declare that they have no conflicts of interest.

\section{Authors' Contributions}

All authors contributed equally to this paper.

\section{Acknowledgments}

Juan A. Aledo was supported by Junta de Comunidades de Castilla-La Mancha under the Grant FEDER SBPLY/17/ 180501/000493. Jose C. Valverde was supported by FEDER OP2014-2020 of Castilla-La Mancha (Spain) under the Grant 2020-GRIN-29225 and by the Ministry of Science,
Innovation and Universities of Spain under the Grant PGC2018-097198-B-I00.

\section{References}

[1] K.-I. Funahashi and Y. Nakamura, "Approximation of dynamical systems by continuous time recurrent neural networks," Neural Networks, vol. 6, no. 6, pp. 801-806, 1993.

[2] A. Khanafer, T. Basar, and B. Gharesifard, "Stability properties of infected networks with low curing rates," in Proceedings of the 2014 American Control Conference (ACC), pp. 3579-3584, IEEE, Portland, OR, USA, June 2014.

[3] S. R. Etesami and T. Başar, "Complexity of equilibrium in competitive diffusion games on social networks," Automatica, vol. 68, pp. 100-110, 2016.

[4] O. C. Imer, S. Yüksel, and T. Başar, "Optimal control of LTI systems over unreliable communication links," Automatica, vol. 42, no. 9, pp. 1429-1439, 2006.

[5] C. Gershenson, "Classification of random boolean networks," "Classification of random boolean networks," in Artifcial Life VIII 1-8, R. K. Standish, M. Bedau, and H. Abbass, Eds., MIT Press, Cambridge, MA, USA, 2002.

[6] C. Gershenson, "Introduction to random boolean networks," 2004, https://arxiv.org/abs/nlin/0408006.

[7] S. A. Kauffman, "Metabolic stability and epigenesis in randomly constructed genetic nets," Journal of Theoretical Biology, vol. 22, no. 3, pp. 437-467, 1969.

[8] S. A. Kauffman, The Origins of Order: Self Organization and Selection in Evolution, Oxford University Press, Oxford, UK, 1993.

[9] L. Raeymaekers, "Dynamics of boolean networks controlled by biologically meaningful functions," Journal of Theoretical Biology, vol. 218, no. 3, pp. 331-341, 2002.

[10] R. Thomas, "Boolean formalization of genetic control circuits," Journal of Theoretical Biology, vol. 42, no. 3, pp. 563-585, 1973.

[11] R. Thomas and R. Dari, Biological Feedback, CRC Press, Boca Raton, FL, USA, 1990.

[12] A. Adiga, C. J. Kuhlman, M. V. Marathe, H. S. Mortveit, S. S. Ravi, and A. Vullikanti, "Graphical dynamical systems and their applications to bio-social systems," International Journal of Advances in Engineering Sciences and Applied Mathematics, vol. 11, no. 2, pp. 153-171, 2019.

[13] N. L. Ackerman and C. E. Freer, "Graph turing machines. Logic, language, information, and computation," in Lecture Notes in Computer Science, pp. 1-13, Springer, Berlin, Germany, 2017.

[14] C. Defant, "Binary codes and period-2 orbits of sequential dynamical systems," Discrete Mathematics \& Theoretical Computer Science, vol. 19, no. 3, p. 12, 2017.

[15] L. B. Kier and P. G. Seybold, "Cellular automata modeling of complex biochemical systems,"“Cellular automata modeling of complex biochemical systems," in Encyclopedia of Complexity and Systems Science, R. A. Meyers, Ed., pp. 848-865, Springer, New York, NY, USA, 2009.

[16] L. B. Kier, P. G. Seybold, and C.-K. Cheng, Modeling Chemical Systems Using Cellular Automata, Springer, New York, NY, USA, 2005.

[17] S. D. Cardell and A. Fúster-Sabater, "Binomial representation of cryptographic binary sequences and its relation to cellular automata," Complexity, vol. 2019, Article ID 2108014, 13 pages, 2019. 
[18] A. Fúster-Sabater and P. Caballero-Gil, "On the use of cellular automata in symmetric cryptography," Acta Applicandae Mathematicae, vol. 93, no. 1-3, pp. 215-236, 2006.

[19] G. Cattaneo, M. Comito, and D. Bianucci, "Sand piles: from physics to cellular automata models," Theoretical Computer Science, vol. 436, pp. 35-53, 2012.

[20] B. Chopard and M. Droz, Cellular Automata for Modeling Physics, Cambridge University Press, Cambridge, MA, USA, 1998.

[21] O. Colón-Reyes, R. Laubenbacher, and B. Pareigis, "Boolean monomial dynamical systems," Annals of Combinatorics, vol. 8, no. 4, pp. 425-439, 2005.

[22] J. Demongeot, E. Goles, M. Morvan, M. Noual, and S. Sené, "Attraction basins as gauges of the robustness against boundary conditions in biological complex systems," PLoS One, vol. 5, no. 8, Article ID e11793, 2010.

[23] J. J. Hopfield, "Neural networks and physical systems with emergent collective computational abilities," Proceedings of the National Academy of Sciences, vol. 79, no. 8, pp. 25542558, 1982.

[24] H. S. Mortveit and C. M. Reidys, "Discrete, sequential dynamical systems," Discrete Mathematics, vol. 226, no. 1-3, pp. 281-295, 2001.

[25] M. Noual, D. Regnault, and S. Sené, "About non-monotony in boolean automata networks," Theoretical Computer Science, vol. 504, pp. 12-25, 2013

[26] É. Remy, P. Ruet, and D. Thieffry, "Graphic requirements for multistability and attractive cycles in a boolean dynamical framework," Advances in Applied Mathematics, vol. 41, no. 3, pp. 335-350, 2008.

[27] J. A. Aledo, S. Martínez, and J. C. Valverde, "Parallel dynamical systems over directed dependency graphs," Applied Mathematics and Computation, vol. 219, no. 3, pp. 1114-1119, 2012.

[28] J. A. Aledo, S. Martinez, F. L. Pelayo, and J. C. Valverde, "Parallel discrete dynamical systems on maxterm and minterm boolean functions," Mathematical and Computer Modelling, vol. 55, no. 3-4, pp. 666-671, 2012.

[29] J. A. Aledo, S. Martinez, and J. C. Valverde, "Parallel dynamical systems over special digraph classes," International Journal of Computer Mathematics, vol. 90, no. 10, pp. 20392048, 2013.

[30] J. A. Aledo, S. Martinez, and J. C. Valverde, "Updating method for the computation of orbits in parallel and sequential dynamical systems," International Journal of Computer Mathematics, vol. 90, no. 9, pp. 1796-1808, 2013.

[31] J. A. Aledo, S. Martinez, and J. C. Valverde, "Parallel discrete dynamical systems on independent local functions," Journal of Computational and Applied Mathematics, vol. 237, no. 1, pp. 335-339, 2013.

[32] J. A. Aledo, S. Martinez, and J. C. Valverde, "Graph dynamical systems with general boolean states," Applied Mathematics \& Information Sciences, vol. 9, no. 4, pp. 1803-1808, 2015.

[33] J. A. Aledo, L. G. Diaz, S. Martinez, and J. C. Valverde, "On the periods of parallel dynamical systems," Complexity, vol. 2017, Article ID 7209762, 6 pages, 2017

[34] J. A. Aledo, L. G. Diaz, S. Martinez, and J. C. Valverde, "Maximum number of periodic orbits in parallel dynamical systems," Information Sciences, vol. 468, pp. 63-71, 2018.

[35] H. S. Mortveit and C. M. Reidys, An Introduction to Sequential Dynamical Systems, Springer, New York, NY, USA, 2007.

[36] J. A. Aledo, L. G. Diaz, S. Martinez, and J. C. Valverde, "On periods and equilibria of computational sequential systems," Information Sciences, vol. 409-410, pp. 27-34, 2017.
[37] J. Aracena, "Maximum number of fixed points in regulatory boolean networks," Bulletin of Mathematical Biology, vol. 70, no. 5, pp. 1398-1409, 2008.

[38] J. Aracena, J. Demongeot, and E. Goles, "Fixed points and maximal independent sets in AND-OR networks," Discrete Applied Mathematics, vol. 138, no. 3, pp. 277-288, 2004.

[39] J. Aracena, A. Richard, and L. Salinas, "Maximum number of fixed points in AND-OR-NOT networks," Journal of Computer and System Sciences, vol. 80, no. 7, pp. 1175-1190, 2014.

[40] J. Aracena, A. Richard, and L. Salinas, "Number of fixed points and disjoint cycles in monotone boolean networks," SIAM Journal on Discrete Mathematics, vol. 31, no. 3, pp. 1702-1725, 2017.

[41] J. Aracena, A. Richard, and L. Salinas, "Fixed points in conjunctive networks and maximal independent sets in graph contractions," Journal of Computer and System Sciences, vol. 88, pp. 145-163, 2017.

[42] A. A. Melkman, T. Tamura, and T. Akutsu, "Determining a singleton attractor of an AND/OR boolean network in $\mathrm{O}$ $\left(1.587^{n}\right)$ time," Information Processing Letters, vol. 110, no. 1415, pp. 565-569, 2010.

[43] A. Richard, "Fixed points and connections between positive and negative cycles in boolean networks," Discrete Applied Mathematics, vol. 243, pp. 1-10, 2018.

[44] F. Robert, "Discrete iterations: a metric study,"in SpringerSeries in Computational Mathematics,Vol. 6, Springer, Berlin, Germany, 1986.

[45] A. Veliz-Cuba and R. Laubenbacher, "On the computation of fixed points in boolean networks," Journal of Applied Mathematics and Computing, vol. 39, no. 1-2, pp. 145-153, 2012.

[46] T. Akutsu, S. Kuhara, O. Maruyama, and S. Miyano, “A system for identifying genetic networks from gene expression patterns produced by gene disruptions and overexpressions," Genome Informatics. Workshop on Genome Informatics, vol. 9, pp. 151-160, 1998.

[47] M. Milano and A. Roli, "Solving the satisfiability problem through boolean networks. AI*IA 99: advances in artificial intelligence (Bologna)," in Proceedings of the 6th Congress of the Italian Association for Artificial Intelligence on Advances in Artificial Intelligence, pp. 72-83, Bari, Italy, September 1792.

[48] F. Bridoux, N. Durbec, K. Perrot, and A. Richard, "Complexity of maximum fixed point problem in boolean networks," Lecture Notes in Computer Science, Vol. 11558, Springer, Berlin, Germany, 2019. 